Max-Planck-Institut für demografische Forschung

Max Planck Institute for Demographic Research

Konrad-Zuse-Strasse 1 - D-18057 Rostock - GERMANY

Tel +49 (0) 3812081 - 0; Fax +49 (0) 3812081 - 202;

http://www.demogr.mpg.de

MPIDR WORKING PAPER WP 2015-007

NOVEMBER 2015

\title{
Renewal and stability in populations structured by remaining years of life
}

Tim Riffe (riffe@demogr.mpg.de)

This working paper has been approved for release by: Mikko Myrskylä (sekmyrskyla@demogr.mpg.de), Head of the Laboratory of Population Health and Head of the Laboratory of Fertility and Well-Being.

(C) Copyright is held by the authors.

Working papers of the Max Planck Institute for Demographic Research receive only limited review. Views or opinions expressed in working papers are attributable to the authors and do not necessarily reflect those of the Institute. 


\title{
Renewal and stability in populations structured by remaining years of life
}

\author{
Tim Riffe \\ Max Planck Institute for Demographic Research
}

November 10, 2015

Research reported in this manuscript was supported by the U.S. National Institute On Aging of the National Institutes of Health under award numbers R01-AG011552 and R01-AG040245. The content is solely the responsibility of the author and does not necessarily represent the official views of the funding agency. 


\section{Structured abstract}

\section{Background}

The Lotka-Leslie renewal model is the core of formal demography. This model is structured by chronological age, and it does not account for thanatological age.

\section{Objective}

I derive a specification of the classic renewal equation that is structured by thanatological age rather than by chronological age. I give both continuous and discrete variants of the derived model, and relate these to the Lotka-Leslie renewal model.

\section{Results}

In stability, the thanatological and chronological renewal models are commensurable, implying identical intrinsic growth rates. I demonstrate approximate symmetry between chronological and thanatological age structure in stability when subject to intrinsic growth rates equal magnitude and opposite sign.

\section{Conclusions}

Birth-death renewal processes can be expressed as death-birth processes, and vice versa.

\section{Contribution}

The thanatological renewal model offers a new perspective on population renewal, and it is valid more generally as an aspect of birth-death processes. 


\section{Introduction}

Contemporary demography is built upon a small set of empirical regularities with respect to chronological age patterns. The models and methods of demography are in varying degrees prefaced on such regularity. There is evidence that many demographic phenomena are best described in the aggregatate as a function of time since birth, and others of time until death, while others can be a function of both to some degree (Riffe et al. 2015, Wolf et al. 2015). This observation motivates incorporating this aspect time-to-death into formal demographic methods and models.

In this paper, I explore some formal demographic consequences of a particular redefinition of age. Instead of counting age as the time passed since birth, consider age as the amount of time left until death. The first kind of age is called chronological age, and the second kind of age is called thanatological age. ${ }^{1}$ Individuals in this case move in the same direction along imaginary life lines, but the reference point for thanatological age is at the end of the life line instead of at the beginning. For individuals, the timing of events in life is exactly identified using either of the two definitions of age, but aggregate patterns differ.

The renewal model relates fertility, mortality, and population structure in a parsimonious form, revealing the long term consequences of a particular set of conditions. All diverse lines of contemporary demographic research are relatable to the renewal model, and by extention to stable population theory. The purpose of this paper is to describe the Lotka-Leslie renewal model when structured by thanatological age. To present the renewal model based on thanatological age is not necessarily a suggestion for a better way to model or project population. Rather, the thanatological renewal model represents

\footnotetext{
${ }^{1}$ This quantity is referred to as residual lifetime in the reliability literaure, or just time-to-death (TTD) elsewhere.
} 
an alternative and coherent way to describe the process of population renewal by relating renewal to a death flow rather than to a birth flow. I have described a limited case of this perspective on renewal for the case of stationary populations (Riffe 2015), and here provide a broader treatment allowing for growth in the population. The chronologicalage classified renewal model and the thanatological renewal model both describe the movement of population stocks over time, but from opposite vantage points.

I couch the present inquiry in terms of aggregate renewal of human populations, but the renewal model is more generally a description of the birth-death process (Cox 1962, Feller 1941). As such, the thanatological renewal model may be applicable in myriad other areas, such as queueing, infectious disease processes, or aspects of renewable aggregates in general. Given the breadth of birth-death models and the speculative applicability of the thanatological renewal model, I am content for the present inquiry to establish the validity of the model. Perhaps there exists a birth-death process for which modelling as a death-birth process makes the most sense. In order to establish the soundness of this model as a description of the human renewal process would require considerable empirical groundwork - It would imply demonstrating that the thanatological age pattern of fertility is empirically regular, and that the time-to-death structure of the population is empirically regular. Such an endeavor would be misplaced, as the objective of this inquiry is not to improve the practice of empirical demography. Instead the objective is to deepen our understanding of the Lotka-Leslie renewal model. The chronological and thanatological renewal models imply one another in the state of stability. We therefore present this model as a property implied by the Lotka-Leslie renewal model, as two sides of the same coin. The result is however more general than that of literal birth and death in human populations.

The next section of this article describes the lifetable method of transforming age- 
classified data into thanatological age classes. I then derive the continuous version of the thanatological renewal model, and some other stable properties. This is followed by the matrix version of the model, which is explained alongside the lifecycle graph. Finally, I explore patterns in stable age structure compared between the two models. I conclude that thanatological renewal offers a new perspective on the familiar Lotka-Leslie model.

\section{Requisites of a thanatological renewal model}

The Lotka-Leslie renewal model (Leslie 1945, Sharpe and Lotka 1911) is situated in a unisex homogenous population framework. The model expressed in continous mathematics contains a component for survival, $\ell(a)$, a scaling factor for growth, $e^{-r a}$, and a component for fertility, $m(a)$ :

$$
1=\int_{a=0}^{\infty} l(a) e^{-r a} m(a) \mathrm{d} a
$$

Each of these elements is structured by chronological age, $a$. The model can be approximated by observed data because chronological age is typically known. The survival function, $\ell(a)$, derives from a lifetable, usually based on age-specific event-exposure mortality rates, which require counts of decedents and an estimate of the population exposed to risk in the same chronological age classes. Chronological age in the growth factor, $e^{-r a}$ pertains to the passage of time more than it does to chronological age (these are synchronous for birth cohorts). The product of this growth factor and $\ell(a)$ gives a function proportional to the stable age structure, $c(a)$, and it is often easier to imagine these two elements together as one for this reason. Female fertility rates, $m(a)$, are based on 
birth counts classified by age-of-mother, and the same variety of exposure to risk. For the two essential rate componenents underlying the renewal equation (1), classification by chronological age is essentially given - it is deterministic in the sense that time passed since birth is typically known by individuals. The kinds of errors in such classification are mostly due to innumeracy, rounding, exaggeration, and similar kinds of biases, but even in such cases, chronological age is fundamentally knowable.

Thanatological age is different in this respect because we do not know when we will die - it is only knowable retrospectively or probabilistically. Under a set of simple assumptions, the same lifetable used to estimate $\ell(a)$ can be used to partition and recombine counts observed by chronological age into estimates classified by thanatological age. Our objective is then to estimate an age structure component, similar to $\ell(a) e^{-r a}$, and a fertility component simialr to $m(a)$, but each for the thanatological case. Taken together, these componenents will yield a thanatological renewal model.

\section{A lifetable approximation of thanatological age}

In general, to make a lifetable-based projective statement about the remaining time distribution of a current stock of population, it is best to to account for population subgroups with different mortality and to account for uncertainty about future changes in mortality. The present inquiry approximates thanatological age using lifetable-projected remaining lifetime, which is intended to approximate remaining lifetime under the strong assumptions of homogenous populations und fixed vital rates. Under these assumptions, each birth cohort undergoes the same pattern of attrition. In a period setting, this means that we can distribute the remaining lifespans of the population in each age class using the same lifetable. 
The basic transformation to thanatological age proceeds by defining a conditional deaths density function, $f(y \mid a)$. For chronological age zero, $f(y \mid 0)$ is identical to $d(a)$, the lifetable deaths distribution (based on a unity radix). For ages higher than 0, simply condition on survival: ${ }^{2}$

$$
f(y \mid a)=\mu(a+y) \frac{\ell(a+y)}{\ell(a)}
$$

where $\mu(a)$ is the force of mortality.

A convenient discretization of (2) is to simply work with the $d_{a}$ column of the lifetable (here with single ages):

$$
f_{y, a}=\frac{d_{a+y}+d_{a+y+1}}{L_{a}+L_{a+1}}
$$

where $L_{a}$ is lifetable exposure. ${ }^{3}$ One produces in this way a probability density function over future death times for each chronological age, $a$. The $f(y \mid a)$ - weighted average of $y$ for a given $a$ returns the familiar remaining life expectancy column of the lifetable, $e(a)$. Since $1=\int_{0}^{\infty} f(y \mid a) \mathrm{d} y$ for each $a$, this density is useful for decomposing data classified by chronological age, such as the population pyramid, into thanatological age classes. If $P(a)$ is a chronological age-classified population count, we can derive $P(y)$, a thanatological age-classified population count $\mathrm{using}^{4}$

\footnotetext{
${ }^{2}$ In the reliability literature, $f(y \mid a)$ is denoted by $X-t \mid X>t$, where $X$ is the total lifespan and $t$ is the current chronological age attained.

${ }^{3}$ Equation (3) will incur some error depending on the method used to derive $L_{a}$. To remove this error, rescale over the $a$ margin so that each distribution conditioned on $a$ sums to 1 . Working with $\ell_{a}$ rather than $L_{a}$ eliminates error and yields similar results, but is not necessarily better.

${ }^{4} \mathrm{~A}$ different-looking but identical definition is found in Brouard (1989).
} 


$$
P(y)=\int_{0}^{\infty} P(a) f(y \mid a) \mathrm{d} a
$$

Figure 1a shows this exercise for 2010 US data (Human Mortality Database), assuming the 2010 period mortality schedule remains constant. When looking from the chronological age perspective, this decomposition reveals thanatological heterogeneity, but when viewed from the thanatological perspective (Figure 1a) it reveals chronological age heterogeneity and the overall thanatological age profile, the future death flow of the current population. Figure $1 \mathrm{~b}$ shows this recomposition for the same 2010 US data. The outer profile of Figure 1b is $P(y)$, under our assumptions.

Figure 1: 2010 US population structure

(a) Chronological age-structure (years lived) decomposed by thanatological age (years left).

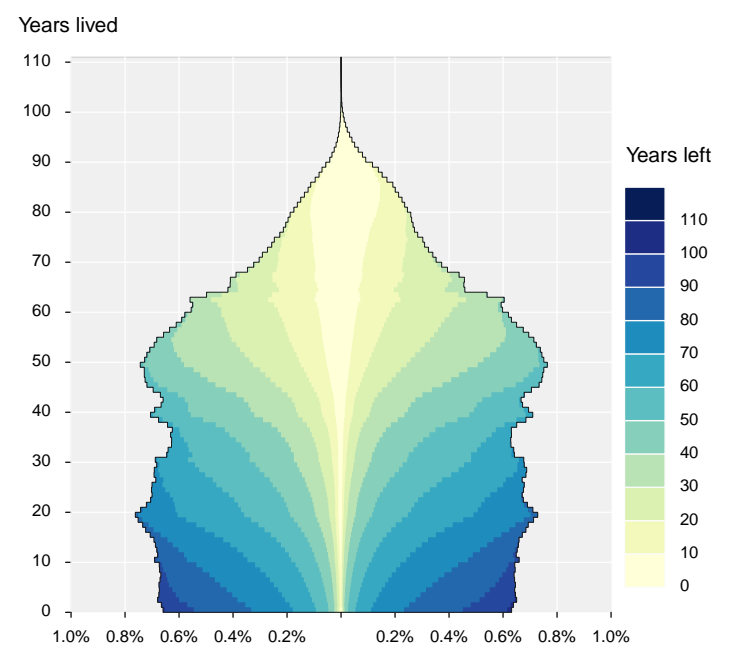

(b) Thanatological age-structure (years left) decomposed by chronological age (years lived).

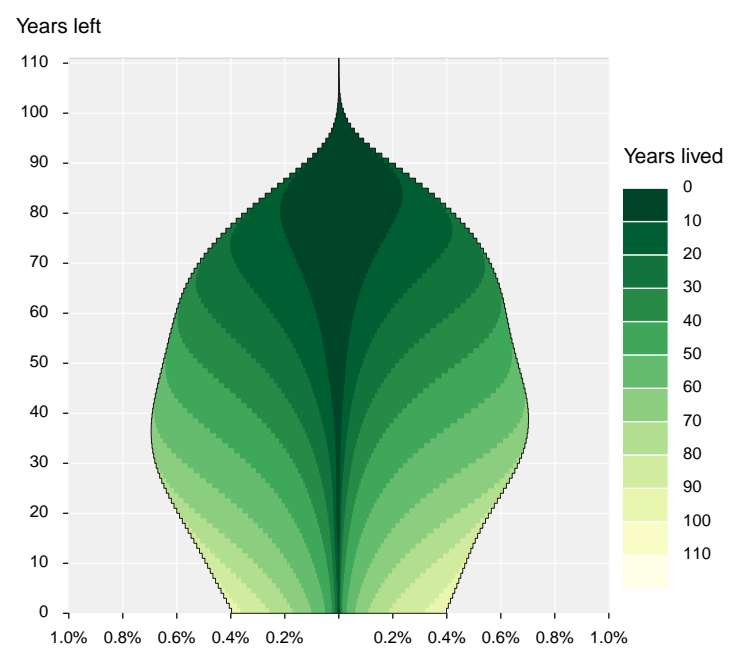

*Population and mortality data from the HMD.

Brouard (1986) applied this method to highlight regional differences in French age 
structure, at times combining this projective method with historical stocks. Brouard (1989), and Vaupel (2009), showed that in stationary populations the marginal chronological and thanatological age structures are identical, and this provides a limited basis for comparison between the two perspectives.

Lifetable-derived thanatological age structure is probabilistic until after death in several senses. In this case, note that counts from a given age class are distributed over a range of higher ages according to a given mortality rate schedule; in this case a period mortality pattern is used. As such, Figure $1 \mathrm{~b}$ is projective in nature, a forward-looking glance at population attrition given the population stock and lifetable of a particular moment, whereas Figure 1a is reflective in nature, since a population's chronological age-structure is the fruit of past demographic forces.

\subsection{Thanatological fertility rates.}

Any count classified by chronological age can be reclassified as in equation (4) given a pertinent lifetable. To derive thanatological fertility rates under these assumptions, $\gamma(y)$, apply (4) to chronological age-classified birth counts, $B(a)$, to get $B(y)$ and again to exposure-to-risk, $E(a)$, to get $E(y)$ and then divide in the usual way:

$$
\gamma(y)=\frac{B(y}{E(y)}
$$

Figure $2 \mathrm{~b}$ represents the full variety in female thanatological period fertility rates that can be found for all years of data that overlap in the HFD and HMD. ${ }^{5}$ I show $\gamma(y)$

\footnotetext{
${ }^{5}$ There are as of this writing 1933 population-years of overlap between the HMD and HFD, including a wide variety of fertility and mortality combinations.
} 
calculated in this way because it enters into the renewal model here studied. Figure 2a gives ASFR for the same populations and years as a more familiar reference, but note that both the $\mathrm{x}$ and $\mathrm{y}$ axes are different. The shape of thanatological fertility curves is speculative and due to the above-mentioned strong assumptions. Nonetheless it is best not to include such rates into a model blindly, so we offer this schematic overview of some of their characteristics.

Period thanatological fertility rates estimated in this way have a characteristic shape. The distribution is wider than that of chronological fertility rates. The range of postchildbearing lifespans stretches from zero, due e.g., to maternal mortality, to at least $100 .^{6}$ This spaghetti plot includes both fertility booms and busts, as well as some mortality crises (1918, WWII). Some patterns in the data (not highlighted in the figure) include that the left tail (a gauge of how orphan-prone a population is) has tended to fall over time. All populations have shown an increase in both the mean and mode of thanatological age at childbearing over time. Several contemporary populations now show modes of over 60 years. Period thanatological total fertility rates track standard period total fertility rates closely, but tend to be somewhat higher (not shown).

In general, thanatological fertility rates will not be useful for purposes of projection, unless their empirical regularity is stronger than typical ASFR. This is a question I do not seek to answer in the present treatment, but in any case such rates are not susceptible to direct measurement. There are alternative ways of defining thanatological fertility rates, for instance by transforming the birth and population vector from a stable population. As proven in appendix B, thanatological fertility rates derived from a stable population imply a certain symmetry with stable populations from the chronological model.

\footnotetext{
${ }^{6}$ For instance, Jeanne Calment lived for another 100 years after giving birth to her daughter.
} 
Figure 2: Chronological and thantological fertility rates, all 1933 country-year combinations present in both the HFD and HMD.* Note different $\mathrm{x}$ and $\mathrm{y}$ scales.

(a) Chronological period fertility rates.

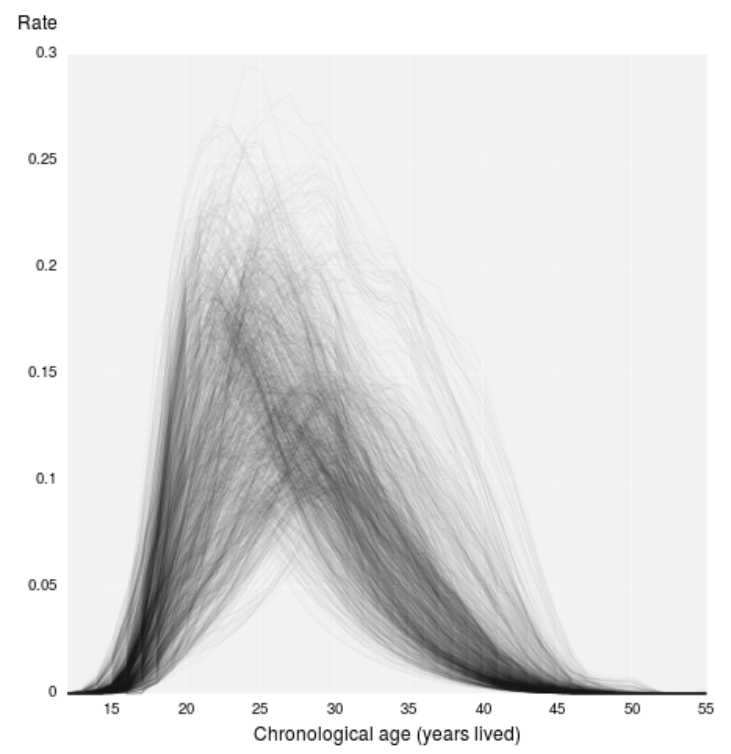

(b) Thanatological period fertility rates.

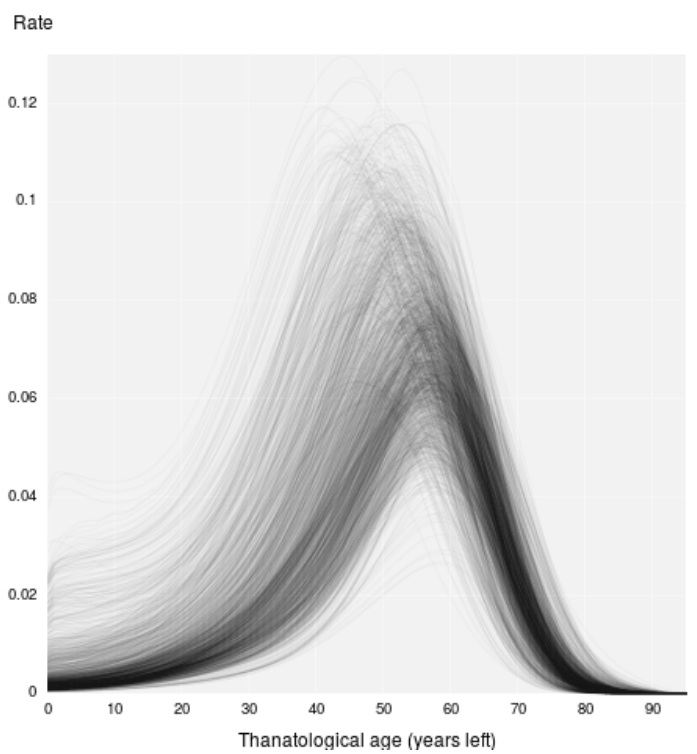

*AUT, 1951-2010; BGR, 1947-2009; BLR, 1964-2012; CAN, 1921-2009; CHE, 1932-2011; CHL, 1992-2005; CZE, 1950-2011; DEUTE, 1956 - 2011; DEUTNP, 1990 - 2011; DEUTW, 1956 - 2011; ESP, 1922 - 2012; EST, 1959 - 2011; FIN, 1939 - 2009; FRATNP, 1946 - 2013; GBR_NIR, 1974 - 2011; GBR_NP, 1974 - 2011; GBR_SCO, 1945 - 2011; GBRTENW, 1938 - 2011; HUN, 1950 - 2009; IRL, 1955 - 2009; ISL, 1963 - 2010; JPN, 1947 - 2012; LTU, 1959 - 2011; NLD, 1950 - 2009; NOR, 1967 - 2009; PRT, 1940 - 2012; RUS, 1959 - 2010; SVK, 1950 - 2009; SVN, 1983 - 2009; SWE, 1891 - 2011; TWN, 1976 - 2010; UKR, 1959 - 2009; USA, 1933 - 2010

\section{The thanatological renewal model}

The renewal model that follows uses thanatological fertility rates, $\gamma(y)$, the form of which is suggested in Figure 2b, and we refer to a homogenous, closed, unisex population based on female vital rates. Assuming constant vital rates, the births for the present year are given by

$$
B(t)=\int_{0}^{\infty} P(y, t) \gamma(y) \mathrm{d} y=\int_{0}^{\infty} P(a, t) m(a) \mathrm{d} a
$$


where $a$ indexes chronological age, $y$ indexes thanatological age, $P(a), P(y)$ are population counts, and $\gamma(y), m(a)$ are exact fertility probabilities (rates), best imagined as the fertility rates of females born to females. The thanatological (first) integral can be broken down back in terms of chronological age:

$$
=\int_{y=0}^{\infty} \int_{a=0}^{\infty} \gamma(y) P(a, t) f(y \mid a) \mathrm{d} a \mathrm{~d} y \quad,
$$

where $f(y \mid a)$ is defined per equation $(2)$, so $d(a) / \ell(0)$. We can relate the present population to past births with $P(a, t)=B(t-a) \ell(a)$ :

$$
=\int_{y=0}^{\infty} \int_{a=0}^{\infty} \gamma(y) B(t-a) d(a+y) \mathrm{d} a \mathrm{~d} y
$$

Eventually strong ergodicity will assert itself, and $B(t)$ will be related to $B(t-a)$ according to a constant factor $e^{r a}$, where $r$ is the familiar intrinsic rate of growth:

$$
=\int_{y=0}^{\infty} \int_{a=0}^{\infty} \gamma(y) B(t) e^{-r a} d(a+y) \mathrm{d} a \mathrm{~d} y
$$

Divide out $B(t)$ to get back a familiar-looking renewal equation:

$$
1=\int_{y=0}^{\infty} \int_{a=0}^{\infty} \gamma(y) d(a+y) e^{-r a} \mathrm{~d} a \mathrm{~d} y
$$

Now compare this to Lotka's chronological formulation in Equation (1), which can be expressed as well as:

$$
1=\int_{a=0}^{\infty} \int_{y=0}^{\infty} m(a) d(a+y) e^{-r a} \mathrm{~d} y \mathrm{~d} a
$$


and note that (11) and (10) are really quite similar, since $\int_{y=0}^{\infty} d(a+y) \mathrm{d} a=l(a)$. For intuition, notice that $l(a)$ is here decomposed into $d(a)$. Imagine a matrix of this decomposition, where one margin is chronological age and the other margin is thanatological age. For the chronological case (1), we multiply chronological age-specific fertility rates, $m(a)$, over the chronological age margin, and for the thanatological case we multiply $\gamma(y)$ over the thanatological age margin.

There are gaps in the above line of development, since the jump from (8) to (9) (strong ergodicity) is assumed for the moment. ${ }^{7}$ The step has some intuition, due to the much greater density of connections within the model; persons from nearly any thanatologcal age can produce offspring that can have any other thanatological age. ${ }^{8}$ In this sense, the smoothing mechanism at play as the population passes through time must be much stronger than that for chronological age, at least in most cases. The mechanisms at play unfold in the same way as those so intuitively described by Arthur (1982), and said proof may apply here without further modification. Later in this paper I note how strong ergodicity is guaranteed given the properties of the thanatolgical projection matrix. A proof of the uniqueness of the solution to (10) is given in Appendix A. A proof that the chronological and thanatological renewal models must yield the same $r$ if the starting population is already stable is given in Appendix B. A fast-converging iterative method for finding $r$ from equation (10) is given in Appendix C

If the thanatological fertility rates do not derive from a stable population, as will typically be the case, then the two age perspectives will, unless by coincidence, yield divergent stable models. There is a strong parallel here with the case of two-sex agespecific fertility rates. As in the case of divergence between male and female single-

\footnotetext{
${ }^{7}$ The step to exponential stability is proven for the discrete version of the model.

${ }^{8}$ I say nearly because the very highest thanatological ages tend to be composed of pre-menarchical females, so these can be thought of as structural zeros.
} 
sex models, there will virtually always be divergence between single-sex models under chronological versus thanatological age, unless the starting population is stable. Even though the modeled population stocks are in a way commensurable, the rates used here, $f(a)$ versus $\gamma(y)$ are calculated on the basis of differently distributed denominators $E(a)$ versus $E(y)$.

Once one finds $r$ from (10), other familiar stable population parameters can be calculated. For instance, we may calculate the mean thanatological generation time, $T^{\star}$ :

$$
T^{\star}=\frac{\int_{y=0}^{\infty} \int_{a=0}^{\infty} y e^{-r a} d(a+y) \gamma(y) \mathrm{d} a \mathrm{~d} y}{\int_{y=0}^{\infty} \int_{a=0}^{\infty} e^{-r a} d(a+y) \gamma(y) \mathrm{d} a \mathrm{~d} y} .
$$

Literally, this is the mean of the remaining lifespans of new mothers in the stable population. The thanatological net reproduction rate, $R_{0}^{\star}$ is related by, e.g.,

$$
R_{0}^{\star}=e^{r T^{\star}}
$$

The birth rate, $b$, is given by

$$
b=\frac{1}{\int_{y=0}^{\infty} \int_{a=0}^{\infty} e^{-r a} d(a+y) \mathrm{d} a \mathrm{~d} y}
$$

the denominator of which is the inside of equation (10) less $\gamma(y)$. This simplifies to

$$
=\frac{1}{\int_{a=0}^{\infty} e^{-r a} l(a) \mathrm{d} a}
$$


which is exactly the same as that valid for chronological age in the Lotka-Euler setup, except that $r$ here comes from the thanatological renewal model. If the stable age structure, $c^{\star}(y)$, is known, then we may take the sum of hypothetical births over thanatological age:

$$
=\int_{y=0}^{\infty} \gamma(y) c^{\star}(y) \mathrm{d} y
$$

The stable thanatological age structure, $c^{\star}(y)$, is the proportion of the stable population with remaining years to live $y$

$$
c^{\star}(y)=b \int_{a=0}^{\infty} e^{-r a} d(a+y) \mathrm{d} a
$$

or, given the stable chronological age structure, $c(a)$, and a lifetable,

$$
\begin{aligned}
& =\int_{a=0}^{\infty} c(a) f(y \mid a) \mathrm{d} a \\
& =\frac{\int_{a=0}^{\infty} d(a+y) e^{-r a} \mathrm{~d} a}{\int_{a=0}^{\infty} l(a) e^{-r a} \mathrm{~d} a} .
\end{aligned}
$$

It has previously been shown that when $r=0$ and $y=a$, that $c(a)=c^{\star}(y)$ (Brouard 1989, Vaupel 2009), and now we can arrive at the same conclusion directly by equating the chronological and thanatological renewal equations. For small non-zero magnitude values of $r$ and survival patterns typical of humans, it is also the case that $c^{\star}(y \mid r) \approx c(a \mid-r)$. This could make a useful heuristic, and the relationship is asymptotically exact as $r$ approaches zero, but the exact relationship is to be found in equation (18). Further stable population quantities may be estimated by similarly translating the various common definitions (e.g., in the glossary of Coale (1972)) to the present perspective. I focus on 
the main model rather than on these.

\section{The thanatological projection matrix}

These descriptions can be made more explicit, and in some ways more tractable, by hashing out the projection matrix that corresponds to the thanatological renewal model. As with the chronological age-structured Leslie matrix, the thanatological projection matrix, $\mathbf{Y}$, is square and of dimension $y \times y$, where $y$ is the number of equal-interval thanatological age classes into which the population is structured. The matrix contains elements for survival and elements for fertility. Unlike Leslie matrices, $\mathbf{Y}$ is not sparse, but is populated primarily with positive entries greater than zero. In the following, I illustrate using a $6 \times 6$ matrix.

Mortality occurs in only the population class with zero remaining years of life. The population of thanatological age 1 in year $t$ moves to 0 in year $t+1$ and dies in the course of that year. Thus, rather than in the subdiagonal, survival elements are located in the superdiagonal. All survival values are 1, since there is no decrement until after living through age 0 . The survival component of $\mathbf{Y}$ is organized as in Matrix 0.1. To imagine a lifecycle graph, each age decrements by one through each lower age in successive order until being absorbed by death after completing thanatological age zero.

Matrix 0.1: Survival component of unisex thanatological projection matrix, $\mathbf{Y}$

$$
\begin{aligned}
& \begin{array}{lllllll}
e_{y} & 0_{t} & 1_{t} & 2_{t} & 3_{t} & 4_{t} & 5_{t}
\end{array} \\
& 0_{t+1}\left(\begin{array}{cccccc}
0 & 1 & 0 & 0 & 0 & 0
\end{array}\right) \\
& 1_{t+1}\left(\begin{array}{llllll}
0 & 0 & 1 & 0 & 0 & 0
\end{array}\right.
\end{aligned}
$$

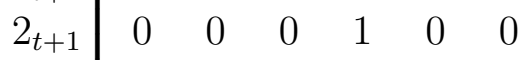

$$
\begin{aligned}
& 3_{t+1} \quad 0 \quad 000000 \\
& 4_{t+1}\left(\begin{array}{llllll}
0 & 0 & 0 & 0 & 0 & 1 \\
0 & 0 & 0 & 0 & 0 & 0
\end{array}\right)
\end{aligned}
$$


Fertility inputs to the matrix are derived from single-sex thanatological fertility and the lifetable $d_{a}$ distribution, where $a$ indexes chronological age and is equated to $y$ remaining years of life for members just born. It is simpler to imagine $d_{a}$ in this case as indexing the future lifespan distribution of newborns (Riffe 2015). Fertility in a thanatologically structured human population occurs in all but the very highest thanatological age classes, which can be thought of as structural zeros for simplicity, as they only contain pre-menarchical females that will have very long lives.

For our $6 \times 6$ example, say that fertility is observed in thanatological classes $0-4$, while the final class has no fertility, where $\gamma_{y}$ indicates the fertility probability for class $y$ in the year $t$ entering population (in the matrix columns). Each $\gamma_{y}$ is then distributed over rows according to $d_{a}$. The fertility entry in row $m$ and column $n$ of $\mathbf{Y}$ will therefore be $\gamma_{n} \cdot d_{m}$. One can assume that those dying over the course of year $t$ (the first column) are exposed to fertility for half of the year, ${ }^{9}$ and so discount the fertility entry accordingly.

Further, infant mortality, $\gamma_{y} \cdot d_{0}$, located in the first row, must also be discounted, since part of the mortality will occur in the same year $t$ and the rest in year $t+1$. The first row of fertility must be further discounted by a factor, $\lambda$, in order to account for the fact that infant mortality is higher in the lower Lexis triangle than in the upper. Of those infants who die in the first year of life, a proportion equal to $\lambda$ do not survive to December 31st of the calendar year in which they were born. ${ }^{10}$ The fertility component of $\mathbf{Y}$ is then composed as in Matrix 0.2 .

\footnotetext{
${ }^{9}$ One might be tempted to not allow for fertility at all for females dying in year $t$, but recall that fertility is measured in the moment of birth, and not conception.

${ }^{10} \lambda$ can be derived directly from death counts data classified by Lexis triangles. If the demographer does not have information to derive $\lambda$ directly, ad hoc or semidirect methods may be used to assign a reasonable proportion, such as 0.9 for contemporary low mortality populations.
} 
Matrix 0.2: Fertility component of unisex thanatological projection matrix, $\mathbf{Y}$

$e_{y}$
$0_{t+1}$
$1_{t+1}$
$2_{t+1}$
$3_{t+1}$
$4_{t+1}$
$5_{t+1}$$\left(\begin{array}{cccccc}(1-\lambda) \frac{\gamma_{0} d_{0}}{2} & (1-\lambda) \gamma_{1} d_{0} & (1-\lambda) \gamma_{2} d_{0} & (1-\lambda) \gamma_{3} d_{0} & (1-\lambda) \gamma_{4} d_{0} & 0 \\ \frac{\gamma_{0} d_{1}}{2} & \gamma_{1} d_{1} & \gamma_{2} d_{1} & \gamma_{3} d_{1} & \gamma_{4} d_{1} & 0 \\ \frac{\gamma_{0} d_{2}}{2} & \gamma_{1} d_{2} & \gamma_{2} d_{2} & \gamma_{3} d_{2} & \gamma_{4} d_{2} & 0 \\ \frac{\gamma_{0} d_{3}}{2} & \gamma_{1} d_{3} & \gamma_{2} d_{3} & \gamma_{3} d_{3} & \gamma_{4} d_{3} & 0 \\ \frac{\gamma_{0} d_{4}}{2} & \gamma_{1} d_{4} & \gamma_{2} d_{4} & \gamma_{3} d_{4} & \gamma_{4} d_{4} & 0 \\ \frac{\gamma_{0} d_{5}}{2} & \gamma_{1} d_{5} & \gamma_{2} d_{5} & \gamma_{3} d_{5} & \gamma_{4} d_{5} & 0\end{array}\right)$

The survival and fertility components of $\mathbf{Y}$ add together elementwise, thus the full $6 \times 6$ matrix is composed as in Matrix 0.3 .

Matrix 0.3: A full unisex thanatological projection matrix, $\mathbf{Y}$

\begin{tabular}{|c|c|c|c|c|c|c|}
\hline$e_{y}$ & $0_{t}$ & $1_{t}$ & $\mathbf{Y}=\frac{}{2_{t}}$ & $3_{t}$ & $4_{t}$ & $5_{t}$ \\
\hline $0_{t+1}$ & $(1-\lambda) \frac{\gamma_{0} d_{0}}{2}$ & $(1-\lambda) \gamma_{1} d_{0}+1$ & $(1-\lambda) \gamma_{2} d_{0}$ & $(1-\lambda) \gamma_{3} d_{0}$ & $(1-\lambda) \gamma_{4} d_{0}$ & 0 \\
\hline $1_{t+1}$ & $\frac{\gamma_{0} d_{1}}{2}$ & $\gamma_{1} d_{1}$ & $\gamma_{2} d_{1}+1$ & $\gamma_{3} d_{1}$ & $\gamma_{4} d_{1}$ & 0 \\
\hline $2_{t+1}$ & $\frac{\gamma_{0} d_{2}}{2}$ & $\gamma_{1} d_{2}$ & $\gamma_{2} d_{2}$ & $\gamma_{3} d_{2}+1$ & $\gamma_{4} d_{2}$ & 0 \\
\hline $3_{t+1}$ & $\frac{\gamma_{0} d_{3}}{2}$ & $\gamma_{1} d_{3}$ & $\gamma_{2} d_{3}$ & $\gamma_{3} d_{3}$ & $\gamma_{4} d_{3}+1$ & 0 \\
\hline $4_{t+1}$ & $\frac{\gamma_{0} d_{4}}{2}$ & $\gamma_{1} d_{4}$ & $\gamma_{2} d_{4}$ & $\gamma_{3} d_{4}$ & $\gamma_{4} d_{4}$ & 1 \\
\hline $5_{t+1}$ & $\frac{\gamma_{0} d_{5}}{2}$ & $\gamma_{1} d_{5}$ & $\gamma_{2} d_{5}$ & $\gamma_{3} d_{5}$ & $\gamma_{4} d_{5}$ & 0 \\
\hline
\end{tabular}

Figure 3 depicts the projection matrix $\mathbf{Y}$ in the form of a lifecycle graph. In this graph, each thanatological age is a node, and death is the absorbing state, denoted with an "X". Population members are born into any of the thanatological age classes, and then follow clockwise around the graph on the thick blue paths in order until finally being absorbed into death (the node labeled "X", not included in the projection matrix). The light 
gray paths represent fertility. These originate from any age class that has a fertility rate greater than zero, which for this toy matrix are all age classes except the 5 th (highest). The $\mathrm{y}^{\text {th }}$ age class is subject to the fertility rate $\gamma(y)$, and these births distribute out to all other age classes according to the lifespan distribution from the lifetable, $d_{a}$. Hence, the rate intensity of a fertility path is determined by the fertility rate of the origin age and probability of having a lifespan equal to the order of the destination node. A fraction of the fertility destined for infant mortality equal to $\lambda$ leads straight to the absorbing state of death. Fertility paths from the 4th thanatological age class are highlighted. These correspond to the fifth column of the example matrix $\mathbf{Y}$, plus an additional path direct to death for the fraction of infant mortality prior to December 31st in year $t$. Paths that loop back into the same age class simply indicate those offspring that are destined to die in the same year as their mother. It is perhaps clearer to see from the density of this graph and by recalling that thanatological fertility distributions are relatively wide (see Figure $2 \mathrm{~b}$ ) that there is a high degree of mixing of thanatological age within populations via reproduction - a strong tendency toward stability.

Thanatological age-classes will ideally terminate at the highest value permitted by data. For the data used in this paper, there are 111 total age classes, which translate to 111 total remaining-years classes $(0-110+)$. In practice $\mathbf{Y}$ becomes a $111 \times 111$ matrix, with most entries non-zero (nearly complete connectivity, such as in Figure 3). Construction may appear tedious for this reason. However, note that the bulk of fertility entries can be derived as the outer (tensor) product $d_{a} \otimes f_{y}$, leaving only the first row and first column mortality discounting followed by the addition of the survival superdiagonal. In most statistical programming languages constructing $\mathbf{Y}$ entails only a couple more lines of code than constructing a Leslie matrix.

Thanatological projection matrices may be manipulated using standard matrix tech- 
Figure 3: The thanatological lifecycle graph

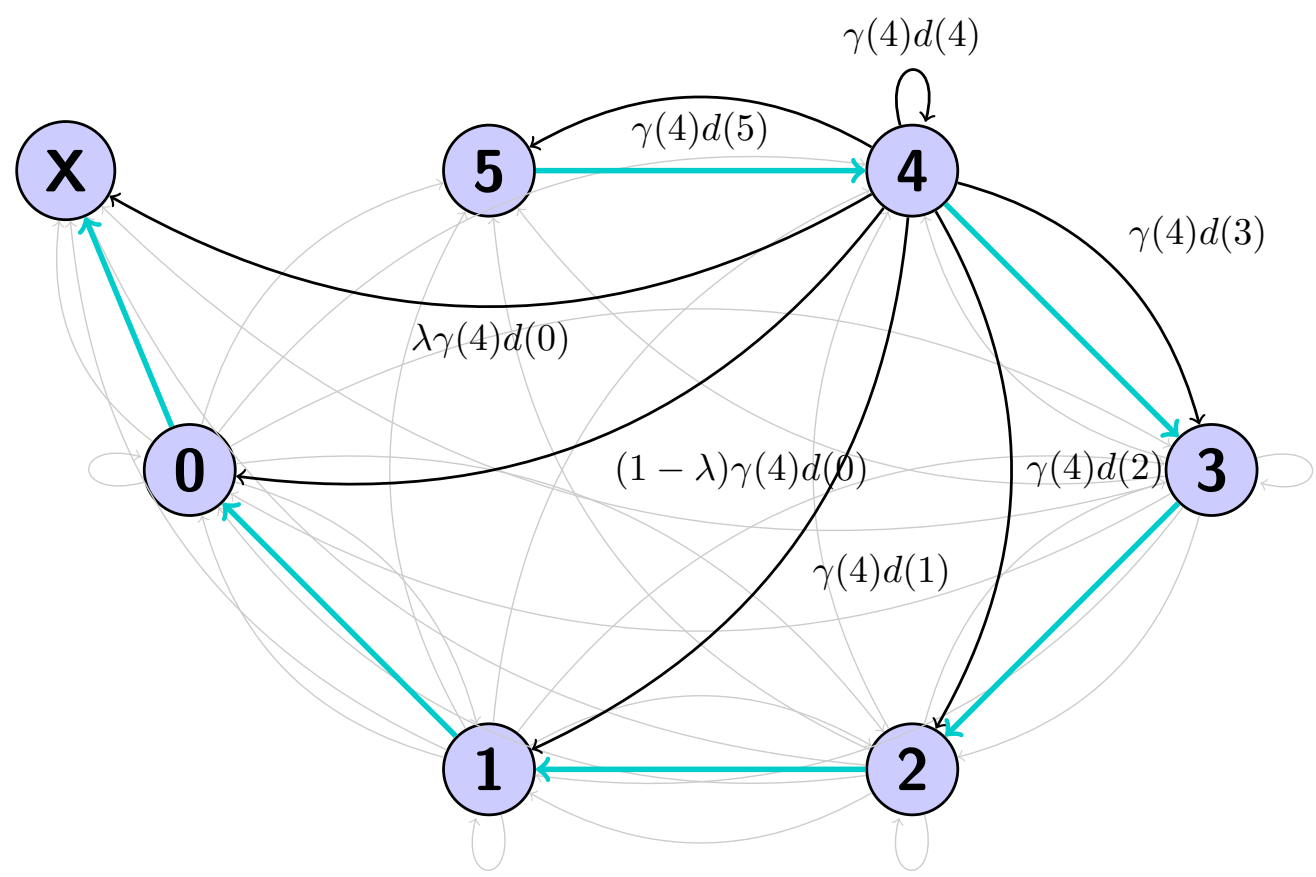

niques applicable to the Leslie matrix. Where $\mathbf{p}$ is a population vector classified by thanatological age, projection proceeds by multiplying $\mathbf{Y}$ from the left: $\mathbf{p}(t+1)=\mathbf{Y} \mathbf{p}(t)$.

For rate schedules typical of human populations, thanatological projection matrices are filled with non-negative values, mostly greater than zero. Raising the matrix to some not-very-large power, $k$, will make all entries greater than zero. This observation, or else by noting that the lifecylce graph is strongly connected, lets us conclude that the matrix is irreducible and primitive. By the Perron-Frobenius theorem, the matrix will always have a unique, dominant, positive real eigenvalue, the natural log of which is the intrinsic growth rate, $r$, and strong ergodicity is assured. In other words, given a fixed matrix and enough time, the population will conform to some stable thanatological age distribution. Strong ergodicity was taken for granted in jumping from equation (8) to (9), but the presently described properties of the matrix model should, albeit unrigorously, 
satisfy lingering uncertainty on that step. The eigenvector corresponding to the dominant eigenvalue gives this stable thanatological age structure. If the fertility rates placed in the matrix are from the stable population, then the growth rate from the thanatological projection matrix is equal to that of the standard Leslie matrix. ${ }^{11}$ The square of the ratio of the 1 st to the 2 nd eigenvalues is the damping ratio, an indicator of the springiness of the stable population structure, $c^{\star}(y)$, if perturbed from the state of stability. ${ }^{12} \mathrm{~A}$ higher damping ratio means that the population structure oscillates back to its stable state faster, i.e., that oscillations decrease in size more rapidly. Thanatological damping ratios are usually higher than chronological damping ratios in the data used elsewhere in this paper.

\section{Stable age structure}

The thanatological stable age structure, as given in equation (17), displays some regular patterns worthy of mention. In Figuree 4 the same underlying mortality pattern, based on US males and females from 2010 (HMD) is rescaled to different mortality levels and growth rates. Figure 4a shows stable age structures by chronological age, while Figure 4b shows stable age structure of the same population by thanatological age. Rows indicate growth rates, descending from positive to negative, while columns indicate the both-sex mean life expectancy, $e(0)$, to which 2010 US mortality is scaled. The rows and columns of Figures $4 \mathrm{a}$ and $4 \mathrm{~b}$ are organized in identical fashion, each element referring to the same intrinsic growth rate and average $e(0)$.

The Brouard-Carey equality is visible by noting that the middle rows from the chrono-

\footnotetext{
${ }^{11}$ Some care needs to be taken in order to demonstrate this point, since common approximations and adjustments used in matrix construction can throw things off. $\mathrm{R}$ code is available from the author to demonstrate this point. See also Appendix B for the corresponding proof from the continuous model.

${ }^{12}$ See Caswell (2001, p.86-87).
} 
logical and thanatological matrices are identical. In comparing any other rows, however, it is as if the thanatological matrix were flipped: a growingy is population in the chronological perspective looks like a shrinking population in the thanatological perspective, and vice versa. While the bottom row of Figure 4 a looks like the top row of Figure 4b, the profiles ar enot rigorously identical, and the symmetry is only noteworthy as rule of thumb. In thinking of the birth flow and the death flow, the approximate reflection of age structure over the $r$-axis does make sense: in a shrinking population new birth cohorts are smaller than old birth cohorts, which means that relatively fewer people are born, and relatively more are in older ages, closer to dying. In this case, the base of the chronological pyramid is relatively thin, while the base of the thanatological leaf is relatively thick. The same holds in reverse for growing populations.

While this heuristic applies to stable populations, but it does not necessarily hold for real observed populations under changing mortality. Further, the observation is only valid for the period perspective in stability. In the cohort perspective, i.e., within closed birth cohorts, the analogy to stationary populations is spot on- That is, within birth cohorts the Brouard-Carey equality is exactly true when applied to the lifespan distribution. 
Figure 4: Stable age structures by life expectancy, $e(0)$, and growth rate, $r{ }^{*}{ }^{*}$ (a) Chronological age structure, $c(a)$.

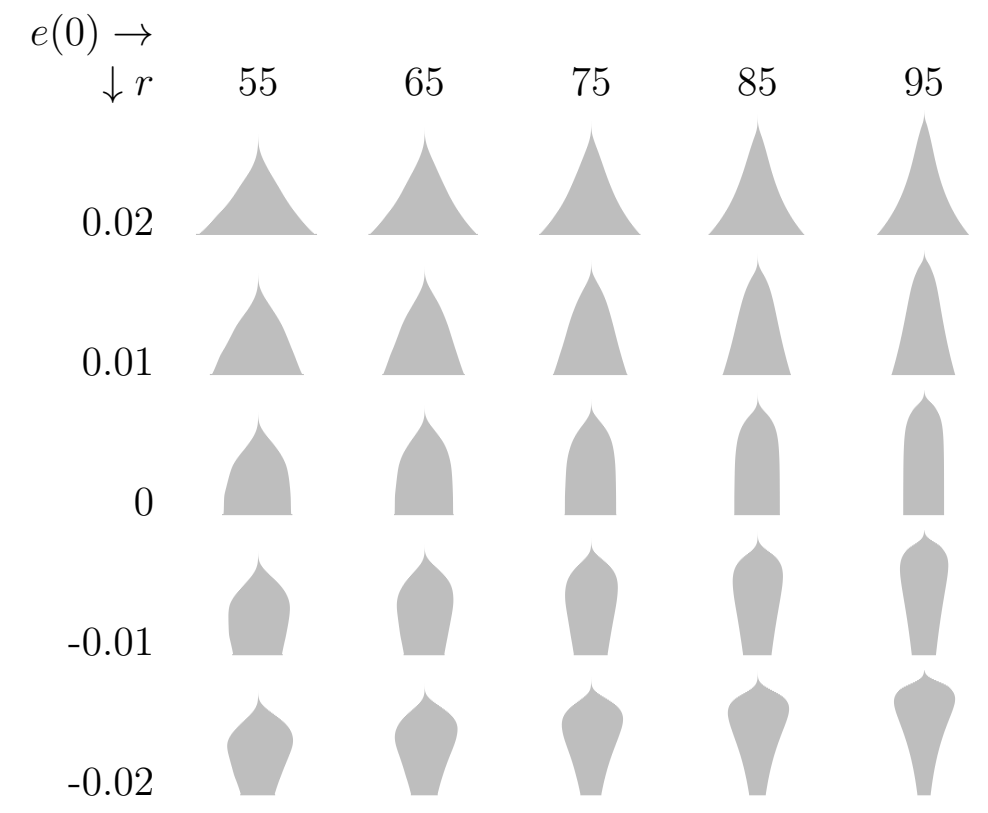

(b) Thanatological age structure, $c^{\star}(y)$.

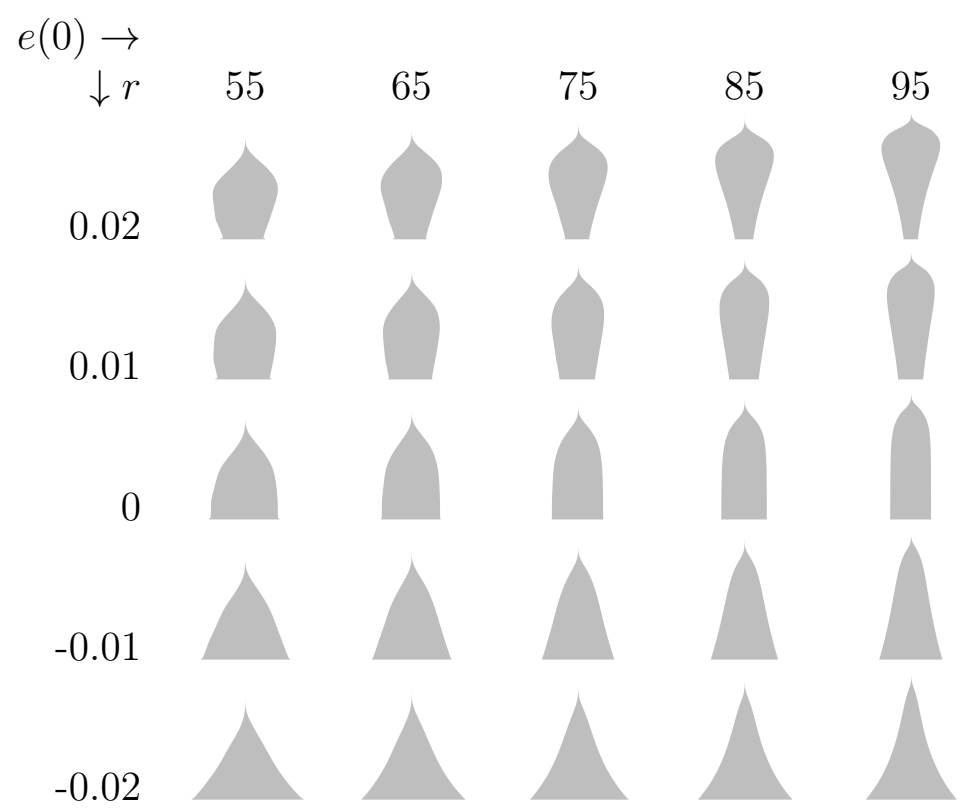

*Underlying mortality pattern based on 2010 USA (HMD) 


\section{Discussion}

The thanatological renewal model is valid, but I do not expect the reader to accept that it is also a sound description of the fundamental forces of population renewal. Specifically, I expect that the fertility rates are best described in the margin by chronological age and not by thanatological age. The main contribution of this inquiry is to demonstrate a transformation of the Lotka-Leslie renewal model, and to highlight a parallel aspect of renewal that occurs irrespective of its empirical regularity. That is, it is the case that in the moment of birth, both progenitor and baby have a certain number of remaining years of life, and that the range of potential values for both is great. The thanatological renewal model therefore is an abstraction of a real process, and not merely an adornment of the chronological renewal model.

What should one imagine under the model of thanatological population renewal? A useful mnemonic bases itself on Figures 1a and 1b. In the chronological age-structured model, new generations appear at the bottom of the pyramid, and move up one class per year. All age-classes are subject to attrition, which is spread out over ages and not readily visible in the pyramid. In the thanatological leaf, each birth cohort increments to the population over the whole range of thanatological age according to $d(a)$, as seen in (10), becoming the shaded layers seen in Figure 1b. Each horizontal step is a death cohort, and these move one step down the pyramid each year without any decrement (indeed incrementing due to births) until reaching the very bottom. In short, the locations of increment and decrement, and the direction of movement (when so visualized) are all swapped. The chronological and thanatological renewal models are almost perfectly opposite descriptions of the same process.

This symmetry is visible in comparing the chronological and thanatological renewal 
equations (11) and (10), and it is also seen in the approximate symmetry between chronological and thanatological age structure profiles under intrinsic growth rates of equal magnitude but opposite sign. The thanatological projection matrix may be thought of as a sort of dual to the Leslie matrix - a twin construct. The thanatological perspective on renewal I present is a hitherto undescribed property of the Lotka-Leslie renewal model. Possible applications of this perspective on renewal are mentioned in Riffe (2015), the only difference in the present inquiry is that we now account for constant growth rates in the population. The present treatment is broader, and potentially applicable to a wider range of birth-death processes. In the first place, we offer a deeper understanding of the Lotka-Leslie renewal models.

\section{A Unique solution for thanatological $r$}

This appendix contains a brief proof that the real solution for the intrinsic growth rate, $r$, is unique for the case of the thanatological renewal model, (10), and can be proven so in essentially the same fashion as those in existence for the Lotka-Euler model, (1). This proof follows that given in Pressat (1973). Define a convenience function, $I(r)$, for the integrand of (10) for a given $r$ and fixed $\gamma(y)$ and $d(a)$ :

$$
I(r)=\int_{y=0}^{\infty} \int_{a=0}^{\infty} \gamma(y) d(a+y) e^{-r a} \mathrm{~d} a \mathrm{~d} y
$$

Since the death distribution function, $d(a)$, and fertility function, $\gamma(y)$, are continuous and non-negative, $\lim _{r \rightarrow+\infty} I(r)=0$ and $\lim _{r \rightarrow-\infty} I(r)=\infty$. If $r_{2}>r_{1}$, then $I\left(r_{1}\right)>I\left(r_{2}\right)$. 
$I()$ is therefore a continuous and monotonically decreasing function of $r$ with boundaries that include the value 1 of (10), and necessarily only obtain this value once. As with the Lotka-Euler equation, there will be more complex conjugate solutions for $r$ in the thanatological model, and these are not explored in this paper.

\section{B Equivalence in stability}

This appendix contains a proof that the thanatological renewal equation implies the same intrinsic growth rate, $r$, as the Lotka-Euler model if the starting population is stable, given a particular set of thanatological fertility rates. This is identical to claiming that the right side of the renewal equation is equal given the same $r$. Since there is a unique real solution, demonstrated in the previous appendix, then it is sufficient to show that the two integral equations are equal under these conditions. The basic relationship is:

$$
\int_{a=0}^{\infty} \ell(a) e^{-r a} f(a) \mathrm{d} a=\int_{y=0}^{\infty} \int_{a=0}^{\infty} d(a+y) e^{-r a} \gamma(y) \mathrm{d} a \mathrm{~d} y
$$

when

$$
\begin{aligned}
\gamma(y) & =\frac{b^{\star}(y)}{c^{\star}(y)} \\
b^{\star}(y) & =\int_{a=0}^{\infty} b(a) \mu(a+y) \frac{\ell(a+y)}{\ell(a)} \mathrm{d} a \\
c^{\star}(y) & =\int_{a=0}^{\infty} c(a) \mu(a+y) \frac{\ell(a+y)}{\ell(a)} \mathrm{d} a
\end{aligned}
$$


Note that:

$$
\begin{aligned}
& c(a)=\frac{\ell(a) e^{-r a}}{\int \ell(a) e^{-r a} \mathrm{~d} a} \\
& b(a)=m(a) c(a)
\end{aligned}
$$

Replacing $\gamma(y)$ with the full expanded expression and plugging into the thanatological renewal equation gives:

$$
=\int_{y=0}^{\infty} \int_{a=0}^{\infty} d(a+y) e^{-r a} \frac{\frac{\int_{t=0}^{\infty} m(t) \ell(t) e^{-r t} \mu(a+y) \frac{\ell(t+y)}{\ell(t)} \mathrm{d} t}{\int \ell(t) e^{-r t} \mathrm{~d} t}}{\frac{\int_{t=0}^{\infty} \ell(t) e^{-r t} \mu(t+y) \frac{\ell(t+y)}{\ell(t)} \mathrm{d} t}{\int \ell(t) e^{-r t} \mathrm{~d} t}} \mathrm{~d} a \mathrm{~d} y
$$

The denominator of $c(a)$ cancels out:

$$
=\int_{y=0}^{\infty} \int_{a=0}^{\infty} d(a+y) e^{-r a} \frac{\int_{t=0}^{\infty} m(t) \ell(t) e^{-r t} \mu(t+y) \frac{\ell(t+y)}{\ell(t)} \mathrm{d} t}{\int_{t=0}^{\infty} \ell(t) e^{-r t} \mu(t+y) \frac{\ell(t+y)}{\ell(t)} \mathrm{d} t} \mathrm{~d} a \mathrm{~d} y
$$

Some $\ell(a)$ 's also cancel out:

$$
=\int_{y=0}^{\infty} \int_{a=0}^{\infty} d(a+y) e^{-r a} \frac{\int_{t=0}^{\infty} m(t) e^{-r t} \mu(t+y) \ell(t+y) \mathrm{d} t}{\int_{t=0}^{\infty} e^{-r t} \mu(t+y) \ell(t+y) \mathrm{d} t} \mathrm{~d} a \mathrm{~d} y
$$

Now $d(a+y)=\mu(a+y) \ell(a+y)$, so we can cancel the inner integral with the denominator:

$$
=\int_{y=0}^{\infty} \int_{a=0}^{\infty} m(a) e^{-r a} \mu(a+y) \ell(a+y) \mathrm{d} a \mathrm{~d} y
$$


Note also that $l(a)=\int_{=a}^{\infty} d(x) \mathrm{d} x$ (present livings are future deaths), which brings us back to the chronological formulation:

$$
=\int_{a=0}^{\infty} e^{-r a} \ell(a) m(a) \mathrm{d} a
$$

This proof establishes that in the stable population there is at least one thanatological fertility rate schedule that will satisfy the constraints of the lifespan distribution and $r$. However, it is not necessary to derive the thanatological fertility distribution in the way prescribed here, and indeed an infinite number of such fertility distributions would satisfy the same constraints. Imagine these fertility rates cross-classified in both age dimensions, where two marginal sums give the thanatological and chronological rates, as they would be calculated over the corresponding cross-classified exposures. The shape of this rate surface can be shifted around in infinitely many ways that would preserve a given growth rate and lifespan distribution, and the above proof shows one such mapping.

\section{An iterative method to find the thanatological $r$}

Coale (1957) proposed a fast-converging iterative approach to estimate the intrinsic growth rate for the Lotka-Euler equation. For the thanatological renewal model, a similar approach may be taken, with some slight modifications to Coale's original. The following steps can be followed to estimate $r$ from Equation (10):

1. Derive a first rough estimate of the mean remaining years of life at reproduction, $\widehat{T^{\star}}$, akin to Lotka's mean generation time, $T$. To start, a good-enough guess is to 
just assume $r=0:^{13}$

$$
\widehat{T^{\star}}=\frac{\int_{y=0}^{\infty} \int_{a=0}^{\infty} y d(a+y) \gamma(y) \mathrm{d} a \mathrm{~d} y}{\int_{y=0}^{\infty} \int_{a=0}^{\infty} d(a+y) \gamma(y) \mathrm{d} a \mathrm{~d} y}
$$

2. A first rough guess at the net reproduction rate, $R_{0}^{\star}$ is given by

$$
R_{0}^{\star}=\int_{y=0}^{\infty} \int_{a=0}^{\infty} d(a+y) \gamma(y) \mathrm{d} a \mathrm{~d} y
$$

3. A first rough estimate of $r, r^{0}$, is given by

$$
r^{0}=\frac{\ln \left(R_{0}^{\star}\right)}{\widehat{T^{\star}}}
$$

4. Plug $r^{0}$ into Equation (10) to calculate a residual, $\delta^{0}$.

5. Use $\delta^{0}$ and $\widehat{T^{\star}}$ to calibrate the estimate of $r$ using

$$
r^{1}=r^{0}+\frac{\delta^{0}}{\widehat{T^{\star}}-\frac{\delta^{0}}{r^{0}}}
$$

6. Repeat step (4) to to derive a new $\delta^{i}$, then step (5) to refine $r^{i}$, until converging on a stable $r$ after some 20 or so iterations, depending on the degree of precision desired $\left(\widehat{T^{\star}}\right.$ is not updated in this process).

The above procedure is more computationally efficient than minimizing the absolute residual of Equation (10) using a generic optimizer. Alternatively, one could use the method of cumulants to estimate $r$, but this would entail less precision than the above (unless one goes beyond, say, five cumulants), and this is not explored.

\footnotetext{
${ }^{13}$ Using the Brouard-Carey equality, the denominator could also be: $\int_{y=0}^{\infty} \ell(y) \gamma(y) \mathrm{d} y$
} 


\section{References}

W Brian Arthur. The ergodic theorems of demography: a simple proof. Demography, 19 (4):439-445, 1982.

Nicolas Brouard. Structure et dynamique des populations. la pyramide des années à vivre, aspects nationaux et exemples régionaux. Espace, populations, sociétés, 4(2): 157-168, 1986.

Nicolas Brouard. Mouvements et modèles de population. Institut de formation et de recherche démographiques, 1989.

H. Caswell. Matrix population models: construction, analysis, and interpretation. Sinaur Associates, Inc. Publishers, 2001.

Ansley J. Coale. A New Method for Calculating Lotka's r-the Intrinsic Rate of Growth in a Stable Population. Population studies, 11(1):92-94, 1957. ISSN 0032-4728.

Ansley J. Coale. The growth and structure of human populations: A mathematical investigation. Princeton University Press Princeton, NJ, 1972.

David Roxbee Cox. Renewal Theory, volume 4. Methuen London, 1962.

Willy Feller. On the integral equation of renewal theory. The Annals of Mathematical Statistics, 12(3):243-267, 1941. 
Human Fertility Database. Max Planck Institute for Demographic Research (Germany) and Vienna Institute of Demography (Austria). online, 2015. Available at www.humanfertility.org (data downloaded on [October 12, 2015]).

Human Mortality Database. University of California, Berkeley (USA) and Max Planck Institute for Demographic Research (Germany). Available at www.mortality.org or www.humanmortality.de (data downloaded October 12, 2015).

Patrick H Leslie. On the use of matrices in certain population mathematics. Biometrika, 33(3):183-212, 1945.

Roland Pressat. L'analyse démographique: concepts, méthodes, résultats. Presses universitaires de France, 3 edition, 1973.

Tim Riffe. The force of mortality by life lived is the force of increment by life left in stationary populations. Demographic Research, 32(29):827-834, 2015.

Tim Riffe, Pil H. Chung, Jeroen Spijker, and John MacInnes. Time-to-death patterns in markers of age and dependency. MPIDR Working Papers, WP-2015(3):25, 2015.

Francis R Sharpe and Alfred J Lotka. L. a problem in age-distribution. The London, Edinburgh, and Dublin Philosophical Magazine and Journal of Science, 21(124):435438, 1911.

James W Vaupel. Life lived and left: Carey's equality. Demographic Research, 20(3): $7-10,2009$.

Douglas A Wolf, Vicki A Freedman, Jan I Ondrich, Christopher L Seplaki, and Brenda C Spillman. Disability trajectories at the end of life: A countdown model. The Journals of Gerontology Series B: Psychological Sciences and Social Sciences, 70(5):gbu182, 2015. 